\title{
Study of the field evaporation mechanism of laser-assisted atom probe
}

\author{
Tetsuo Terakawa,${ }^{1 *}$ Norihito Mayama, ${ }^{1}$ Yasuko Kajiwara and Masanori Owari ${ }^{1,2}$ \\ ${ }^{1}$ Institute of industrial Science, The University of Tokyo, 4-6-1 Komaba, Meguro-ku, Tokyo 153-8505, Japan \\ ${ }^{2}$ Environmental Science Center, The University of Tokyo, 7-3-1 Hongo, Bunkyo-ku, Tokyo 113-0033, Japan \\ *t-tera@iis.u-tokyo.ac.jp
}

(Received : October 3, 2010; Accepted : December 10, 2010)

\begin{abstract}
The three-dimensional atom probe (3DAP) has been used to analyze atomic-scale characterization of a number of nanostructured materials. However, it faces a problem of uncertain field evaporation mechanism by using laser at this time. Resolving this problem is very important to carry out the quantitative analysis in 3DAP. In this paper, we focused on the different reflectivity of tungsten $(50 \%)$, nickel $(70 \%)$ and aluminum $(85 \sim 90 \%)$, and measured them with changed laser power in the 3DAP. From the results, it was indicated that the shift of detection ions with the increase of laser power was different by the reflectivity of material. Also, we compared the field evaporation behavior at the laser pulse with that at the voltage pulse. According to these results, we verified the effect that affected the field evaporation mechanism due to the difference of reflectivity.
\end{abstract}

\section{Introduction}

The electronic devices supporting highly-networked information society are developing rapidly. Therewith, the analysis method which is employed in manufacture and evaluation of these electronic devices are required higher performances. In these analysis methods, the 3DAP has ultimate resolution against individual atoms and enables to visualize three-dimensional structure with atomic scale [1]. In the 3DAP, a specimen of the needle shape is used in the measurement. According to apply high DC voltage, atoms are field evaporated from the needle apex. From the time-of-flight, the atom is identified. In addition, from the hitting position on the detector and the sequences of detection, three-dimensional structure can be reconstructed. In the traditional measurement by using nanosecond voltage pulses, 3DAP had a performance limited only material with high conductivity. However, recent successful implementation of laser pulse to assist field evaporation has expanded the application areas of the atom probe technique to a variety of materials, including semiconductors and insulators.

The laser pulse brought in a lot of advantages with the 3DAP analysis. However, the field evaporation mechanism by using femtosecond laser pulse is still controversial. In the field evaporation mechanism, two types of mechanisms by laser irradiation are speculated. One is the thermal effect [2], the other is the electrical field modulation [3]. Gaining knowledge on the field evaporation mechanism is very important to deduce atomic behavior on a sample surface during the 3DAP analysis.

In our previous work [4], despite aluminum has a lower field evaporation voltage than tungsten, the field-evaporated ions synchronized laser pulse were not observed with the laser power less than $20 \mathrm{~nJ}$, and tungsten ion was field-evaporated less than $1 \mathrm{~nJ}$ laser power. Consequently, it is considered that this change is due to the reflectivity of tungsten $(50 \%)$ and aluminum (85 90\%). The energy absorption efficiency of tungsten is 25 times higher than that of aluminum.

In this paper, we focused on the difference of reflectivity of tungsten, aluminum and nickel $(70 \%)$, and measured them with changed laser power. We report the effect by difference of reflectivity for field evaporation.

\section{Experiment}

Atom probe measurement was carried out in the 3DAP developed in our laboratory [5]. This 
instrument is equipped with a femtosecond pulse laser (1064 nm wavelength, 300 fs duration) and a cryogenerator. Needle specimens for the 3DAP analysis were prepared with metal wires: tungsten, aluminum and nickel. The diameter was $0.1 \mathrm{~mm}$. These wires were electropolished by electropolishing solution appropriate to each material: tungsten $(1 \mathrm{M} \mathrm{NaOH})$, nickel and aluminum $\left(60 \% \mathrm{HClO}_{4}+99.0 \%\right.$ acetone $)$. The applied voltage (2 6 V) was configured relatively high at the start, and let down by degree. Finally, the specimen was cut off at the lowest voltage. The needle apex with the curvature radius of 100 $\mathrm{nm}$ was confirmed with a transmission electron microscope (TEM).

The specimen was mounted on the sample holder with the local electrode and set in to the 3DAP [6,7]. The electrode was made by platinum and has a hole with 200 um diameter. The measurement was carried out under the cooling condition $(\sim 60 \mathrm{~K})$. Laser power was reduced to $0.6,1.2,2.5$ and $5.0 \mathrm{~nJ}$ by using power cut filter from maximum power of $25 \mathrm{~nJ}$. The spot size of this laser was $20 \mu \mathrm{m}$, and the laser was adjusted to irradiate the specimen apex. The sample was measured at the above laser powers in the order of increasing laser power. The standing voltage applied to the needle specimens was as follows: tungsten $(1.8 \sim 2.4 \mathrm{kV})$, nickel $(1.2 \sim 1.9 \mathrm{kV})$ and aluminum $(3.0 \sim 4.4 \mathrm{kV})$.

\section{Results and Discussion}

The mass spectra of tungsten, nickel and aluminum measured at different laser power were shown in Fig. 1. These spectra were normalized by the maximum intensity of each spectrum. In the spectra of tungsten (a) and nickel (b), some peaks were observed at $0.6 \mathrm{~nJ}$ laser power. On the other hand, any peak could not be detected in the spectrum of aluminum (c) at less than $1.2 \mathrm{~nJ}$ laser power. In addition, in the spectra of tungsten and nickel, the intensity of the multiply charged ions was decreased along with increase of laser power. This phenomenon was not observed in the case of aluminum. These results indicate that aluminum is not affected efficiently by laser irradiation in comparison with tungsten and nickel. This corresponds to the fact that the energy absorption efficiency of tungsten is 25 times higher than that of aluminum.

Figure 2 shows the field evaporation image of tungsten sample measured at different laser power. The crystal graphic planes were observed at low laser power. However, it jumbled along with increase of the laser power, and could not be
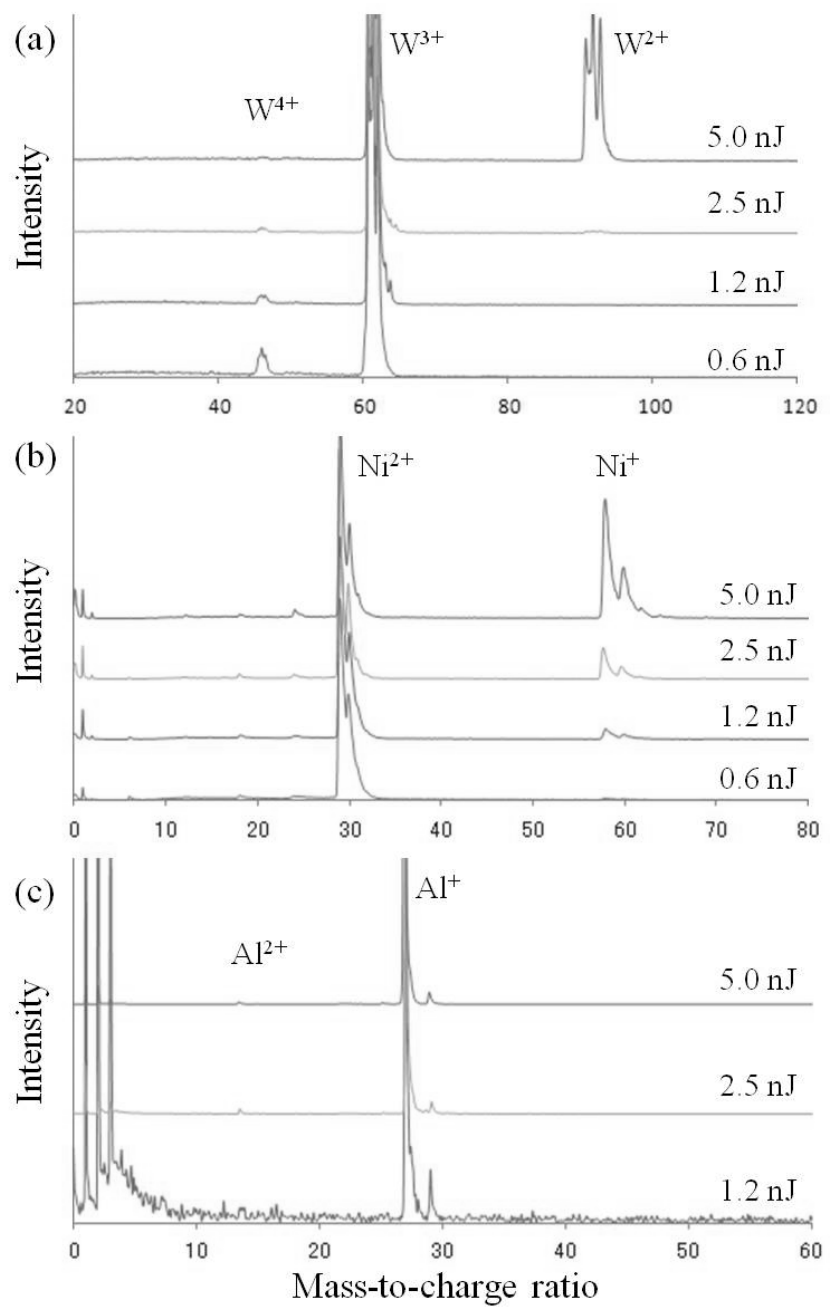

Fig. 1. The mass spectra of tungsten (a), nickel (b) and aluminum (c) with different laser power.

observed eventually. This result can be explained by the thermal effect with laser irradiation. So, the needle apex was warmed by laser irradiation and localized ions were induced the thermal vibration. As a result, the atoms could not fasten at a point and the crystal plane could not be observed clearly.

Figure 3 shows the spectra obtained by using voltage pulse. From this figure, the intensity of $\mathrm{Al}_{2}{ }^{3+}$ peak increases along with applied voltage (standing voltage + voltage pulse). The multiply charged ions were detected largely. This is well known phenomenon [8]. Because, the higher voltage is applied, the higher the tunneling probability of electrons occurs. Therefore, more electrons are transferred from ions into the inside of apex. On the other hand, in the spectra of laser pulse, these changes are not observed. If electrical field is affected by laser power, the amount of multiply charged ions should increase with the increasing of laser power. However, the opposite 
change occurred in fact. This result indicates that the effect of electrical field modulation does not depend on the laser power so much.
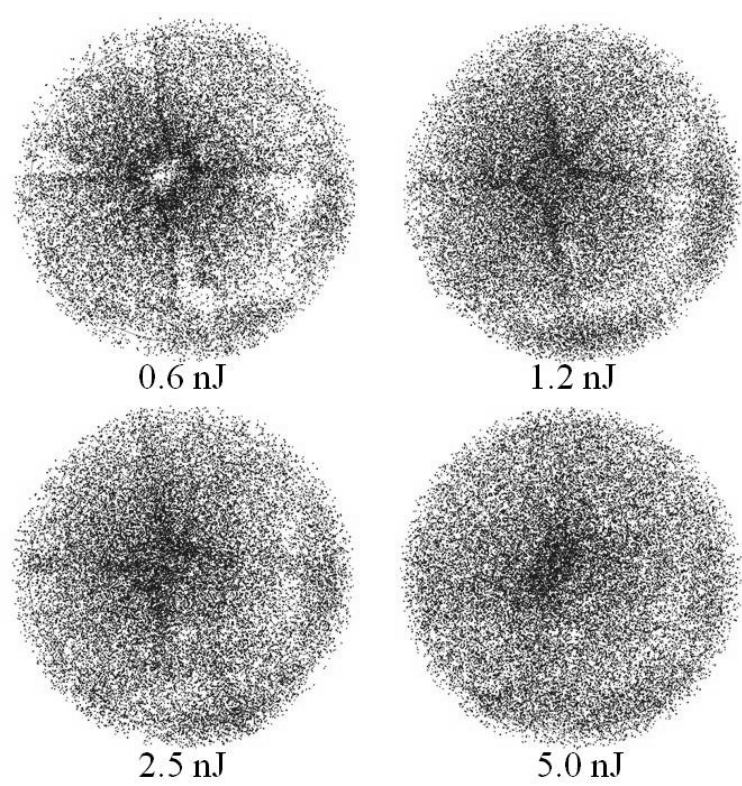

Fig. 2. The field evaporation image of tungsten at different laser power.

\section{Conclusions}

The 3DAP measurement with changed laser power was carried out for tungsten, nickel and aluminum. From these results, only the ion of aluminum origin could not be detected at $0.6 \mathrm{~nJ}$ laser power. Also, from the field evaporation images of tungsten, the field evaporation was subjected to the thermal effect by laser irradiation. These results corresponded to the difference of the energy absorption efficiency determined by the reflectivity of material.

From the comparison with the spectra of aluminum obtained at laser pulse and voltage pulse, the electrical field modulation is not affected by laser power so much.

\section{Acknowledgement}

This work was supported by Grant-in-Aid for Creative Scientific Reserch No. 18GS0204 from Japan Society for the Promotion of Science.

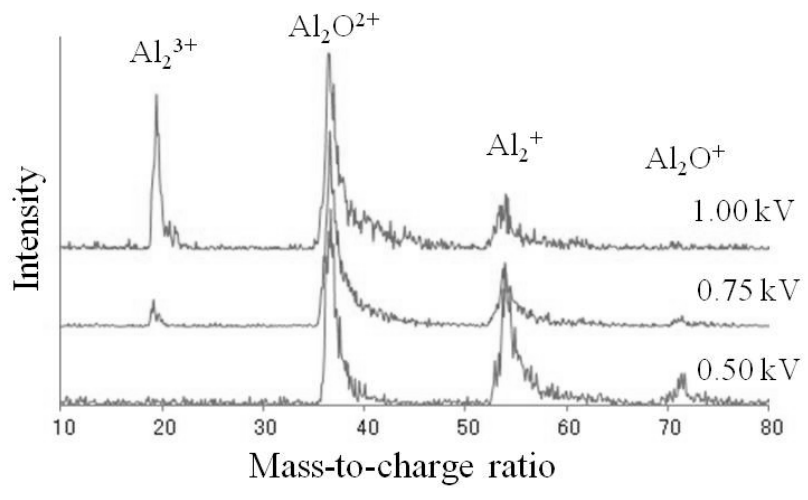

Fig. 3. The spectra of aluminum measured at different voltage pulse (standing voltage is $1.5 \mathrm{kV}$ ).

\section{References}

[1]M. K. Miller, Atom Probe Tomography Analysis at Atomic Level (Kluwer Academic/Plemium Publishers, New York, 2000)

[2] M. K. Miller, Atom Probe Field Ion Microscopy (Oxford University Press, Oxford, 1996).

[3] B. Gault, F. Vurpillot, A. Bostel, A. Menand and B. Deconihout, Appl. Phys. Lett. 86, 094101 (2005)

[4] Y. Kajiwara, N. Mayama, T. Iwata and M. Owari, presented at $57^{\text {th }}$ Spring Meeting of The Japan Society of Applied Physics, 18p-p8-9, Hiratsuka 2010.

[5]N. Mayama, Y. Kajiwara, S. Mikami, S. Ito, T. Kaneko, T. Iwata and M. Owari, J. Surf. Sci. Nanotech. 7, 35-38 (2009).

[6]N. Mayama, C. Yamashita, T. Kaito, M. Nojima and M. Owari, Surf. Interface Anal. 40, 1610 (2008).

[7]S. Ito, T. Kaneko, C. Yamashita, T. Kaito, T. Adachi, T. Iwata, N. Mayama, M. Nojima, M. Taniguchi and M. Owari, Surf. Interface Anal. 40, 1696 (2008).

[8]D. R. Kingham, Surf. Sci. 116, 273 (1982). 